and biphenyl to $1700 \mathrm{~A},{ }^{8}$ it is assumed that the introduction of a methylene bridge (fluorene is $2,2^{\prime}$-methylene-biphenyl) results in an approximately planar structure in which the phenyl groups are not symmetrical about an axis through the $4,4^{\prime}$-positions. This is in agreement with dipole moment measurements which favor this configuration. ${ }^{9}$ The band positions change due to alkyl substitution (bathochromic shift) and the intensities of the ${ }^{1} B_{b}$ bands decrease as is indicated by the decrease in $f$-values. It is to be noted that essentially the same occurs in the comparison of the spectra and the $f$-values of anthracene and phenanthrene.

Similar regularities (bathochromic shifts and decrease in $f$-values) are also observed in the spectra of 2-phenylnaphthalene and its methylene derivative, 1,2-benzfluorene, and in 2-phenylazulene and its methylene derivative, indeno-azulene, as can be seen from the data in Fig. 3 and Table II, although in these

${ }^{8}$ J. R. Platt and H. B. Klevens (unpublished results).

${ }^{9}$ Hughes, LeFevre, and LeFevre, J. Chem. Soc., 202 (1937). compounds the decreases are smaller due probably to less bending of the molecule. There is seen in these compounds only the normal bathochromic effect due to alkyl substitution and not the marked changes in intensity normally associated with twisting of double bonds and twisting of rings from planar positions. The ${ }^{1} C_{b}$ band, transversely polarized to the long axis of the molecule, increases in intensity upon loss of $C_{2 v}$ symmetry about this long axis as is seen in a comparison of the $f$-values of 0.28 for anthracene and 0.60 for phenanthrene. A similar increase is observed for the same transition in 2-phenylazulene and indeno-azulene with $f$-values of 0.41 and 0.55 , respectively, although this increase is also smaller than that observed in the case of the more highly bent polyacene, phenanthrene. The approximated $f$-values of this singlet ${ }^{1} C_{b}(?)$ band in biphenyl and fluorene, and 2-phenylnaphthalene and 1,2-benzfluorene are to be expected to show a similar small increase in going from the linear to the slightly bent methylene derivative.

\title{
On the Notion of Pressure in a Canonical Ensemble
}

\author{
R. J. RIDDELl, JR. AND G. E. Uhlenbeck \\ University of Michigan, Ann Arbor, Michigan
}

(Received April 6, 1950)

\begin{abstract}
Recently a controversy has arisen between J. De Boer and H. S. Green concerning the notion of pressure in a canonical ensemble. According to Green, only classically is the pressure as derived from the partition function equal to that obtained from the virial theorem, while at low temperatures, at which quantum effects become important, there will be considerable deviations between the two. De Boer attempts to prove that the two pressures are actually identical. We have come to the same conclusion, and shall show this in several ways; first, by considering a simple example (Section 2), and then in general using the energy representation (Section 3). We believe that the discrepancy between the two pressures which Green has found is in fact due to improper handling of the effect of the wall of the vessel in which the particles are contained. Finally, we are of the opinion that Green's criticism of De Boer's calculation is not justified, and in the last section arguments are given to show that the traces of all commutators of interest in quantum statistical mechanics are zero.
\end{abstract}

\section{INTRODUCTION}

$\mathrm{R}$ ECENTLY a controversy has arisen between J. De Boer and H. S. Green ${ }^{1}$ concerning the notion of pressure, in a canonical ensemble. According to Green, the pressure as derived from the partition function $Z$ :

$$
p=1 / \beta Z \cdot \partial Z / \partial V,
$$

where $\beta=(k T)^{-1}$, is not necessarily equal to the pressure as derived from the virial theorem:

$$
p V=\frac{2}{3}\left\langle K_{n}\right\rangle_{A v}+\frac{1}{3}\left\langle\Phi_{n}\right\rangle_{A v}
$$

where $K$ is the kinetic energy, $\Phi$ is the virial of the

\footnotetext{
${ }^{1}$ J. De Boer, Physica XV, 843 (1949); Nuovo Cimento Supplement to the Statistical Mechanics Conference at Florence (May, 1949), p. 199 and discussion. H. S. Green, Physica XV, 882 (1949). Compare also J. Yvon, Comptes Rendus 227, 763 (1949).
}

intermolecular forces, and where the $\langle\cdots\rangle_{A v}$ means an average over the canonical ensemble.* Green asserts that the first pressure (which he calls the thermodynamical pressure) is the one exerted by the system in the equilibrium state, while the second (kinetic pressure) is the one occurring in the hydrodynamical equations. ${ }^{2}$ Only in classical statistical mechanics would these two pressures be identical. However, according to Green, as soon as quantum effects become important (as would be the case at very low temperatures) an

* Compare, for instance, Tolman, Principles of Statistical Mechanics, Chapter IX. If $F$ is any kind of operator then in the energy representation:

$$
\left\langle F_{n}\right\rangle_{\mathrm{Av}}=\frac{1}{Z} \Sigma F_{n} \exp \left(-\beta E_{n}\right),
$$

where $F_{n}$ is the average of $F$ in a state of energy $E_{n}$.

${ }^{2}$ H. S. Green, Proc. Roy. Soc. A194, 244 (1949). 
appreciable difference between the two pressures will occur.

On the other hand, De Boer has attempted to show that the two pressures as given by (1) and (2) are alroays identical. Since the argument does not seem to have been completely settled, and since Green's result is so different from the usual conception, a further analysis seems justified. In the following we will confirm De Boer's result using a slight modification of his method of proof. Green's criticism of De Boer's proof cannot, in our opinion, be justified. The origin of the discrepancy between Green's kinetic and thermodynamic pressures lies, in our opinion, in the treatment of the boundary conditions due to the potential of the wall of the vessel in which the system is enclosed. Green (and also De Boer) represents the vessel by a sudden infinite potential jump, and it is well known that with such potentials the formulas of the transformation theory have to be used with caution.

\section{A SIMPLE EXAMPLE}

In order to illustrate in an explicitly soluble case that with a non-singular wall potential the two pressures (1) and (2) are identical, consider the following simple example: two equal one-dimensional particles bound together by a harmonic potential, moving in a "vessel" represented by another, but very weak, harmonic potential. The Hamiltonian for the system is:

$$
\mathfrak{H}=\left(p_{1}{ }^{2}+p_{2}{ }^{2}\right) / 2 m+\frac{1}{2} m \omega^{2}\left(q_{1}{ }^{2}+q_{2}{ }^{2}\right)+\frac{1}{2} m \alpha^{2}\left(q_{1}-q_{2}\right)^{2}
$$

or, introducing the center of gravity and relative coordinates $Q, q$ :

$$
\mathfrak{H C}=P^{2} / 4 m+p^{2} / m+m \omega^{2} Q^{2}+\frac{1}{4} m \omega_{1}^{2} q^{2}
$$

where :

$$
\omega_{1}^{2}=\omega^{2}+2 \alpha^{2} .
$$

Clearly the energy values are:

$$
E_{N, n}=\left(N+\frac{1}{2}\right) \hbar \omega+\left(n+\frac{1}{2}\right) \hbar \omega_{1} .
$$

To find the "pressure" from the partition function $Z=\sum_{N, n} \exp \left(-\beta E_{N, n}\right)$ one has to identify the "volume" of the vessel with $1 / \omega$. To prove, therefore, the equality of the thermodynamic and kinetic pressures, one has to show that:

$$
-\omega^{2} / \beta Z \cdot \partial Z / \partial \omega=\omega\left\langle 2 K_{n}+\Phi_{n}\right\rangle_{A v} .
$$

The factor $\frac{1}{3}$ in (2) must be omitted since the problem is one dimensional.

Because of the harmonicity of the forces one has immediately:

$$
\begin{aligned}
2 K_{N, n} & =E_{N, n} \\
\Phi_{N, n} & =-\sum_{i}\left(q_{i} \frac{\partial V_{\text {int }}}{\partial q_{i}}\right)_{N, n}=-m \alpha^{2}\left(q^{2}\right)_{N, n} \\
& =-\left(n+\frac{1}{2}\right) 2 \hbar \alpha^{2} / \omega_{1} .
\end{aligned}
$$

On the other hand:

$$
\begin{aligned}
-\frac{\omega^{2}}{\beta Z} \cdot \frac{\partial Z}{\partial \omega} & \\
= & \frac{\omega^{2}}{Z} \sum_{N, n} \frac{\partial E_{N, n}}{\partial \omega} \exp \left(-\beta E_{N, n}\right) \\
= & \omega / Z \sum_{N, n}\left\{E_{N, n}-\left(n+\frac{1}{2}\right) 2 \hbar \alpha^{2} / \omega_{1}\right\} \exp \left(-\beta E_{N, n}\right)
\end{aligned}
$$

so that (3) is evident.

\section{THE GENERAL PROOF}

To perform the differentiation of $Z$ with respect to the volume in general, Green and also De Boer use the trick of measuring all coordinates with a unit $L$, which is characteristic of the vessel. In this way they were able to shift the differentiation with respect to the boundary to a differentiation of the operator $\exp (-\beta \mathcal{H})$. To show how this works, we will use as in Section 2 the energy representation of all operators. If $V=L^{3}$, then from (1):

$$
p=-\frac{1}{3 V Z} \sum_{n} L \frac{\partial E_{n}}{\partial L} \exp \left(-\beta E_{n}\right) .
$$

In order to determine $\partial E_{n} / \partial L$ we must go to the Schroedinger equation for the whole system:

$$
\mathfrak{H C}_{n} \equiv\left[K+V_{\text {int }}+W\left(q_{i} / L\right)\right] \Psi_{n}=E_{n} \Psi_{n},
$$

where $V_{\text {int }}$ is the potential of the intermolecular forces and where we have explicitly introduced the potential $W$ due to the wall. It is best to consider $W$ as a smooth, though nearly step-like, function near the boundary, becoming infinite for $q_{i} \rightarrow \pm \infty$. There is then no need to impose a boundary condition on $\Psi_{n}$ (except for the usual one that $\Psi_{n} \rightarrow 0$ as $q_{i} \rightarrow \pm \infty$ ), and all the coordinates $q_{i}$ can be taken from $-\infty$ to $+\infty$. Since $W$ depends on $L, \Psi_{n}$ and $E_{n}$ are also functions of $L$. Now let us replace $q_{i}$ by $L \bar{q}_{i}$, and then differentiate (5) with respect to $L$, considering the $\bar{q}_{i}$ as constant. Thus :

$$
\frac{\partial \mathcal{F}}{\partial L} \Psi_{n}+\mathcal{H C} \frac{\partial \Psi_{n}}{\partial L}=\frac{\partial E_{n}}{\partial L} \Psi_{n}+E_{n} \frac{\partial \Psi_{n}}{\partial L} .
$$

Multiplying on the left by $\Psi_{n}{ }^{*}$ and integrating over all coordinates, one obtains:

$$
\frac{\partial E_{n}}{\partial L}=\int \Psi_{n}^{*} \frac{\partial \mathcal{F} C}{\partial L} \Psi_{n} d \tau,
$$

since the terms in $\left(\partial \Psi_{n} / \partial L\right)$ cancel using the Hermitian property of $\mathcal{H}$. Since, in terms of the $\bar{q}_{i}$ :

$$
\begin{aligned}
\mathfrak{H}=- & \frac{\hbar^{2}}{2 m L^{2}} \sum_{i} \\
& \frac{\partial^{2}}{\partial \bar{q}_{i}^{2}} \\
& +V_{\text {int }}\left(L \bar{q}_{1}, \cdots, L \bar{q}_{N}\right)+W\left(\bar{q}_{1}, \cdots, \bar{q}_{N}\right)
\end{aligned}
$$


in $(\partial \mathfrak{H C} / \partial L)$ the wall potential drops out and one verifies easily:

where

$$
L(\partial \mathcal{H} / \partial L)=-2 K-\Phi
$$

$$
\Phi=-\sum_{i} q_{i} \frac{\partial V_{\text {int }}}{\partial q_{i}}
$$

is the virial of the intermolecular forces. From (6), therefore,

$$
L\left(\partial E_{n} / \partial L\right)=-2 K_{n}-\Phi_{n}
$$

and substituting in (4) gives the virial theorem (2).

\section{DISCUSSION OF GREEN'S CALCULATION}

Green uses the coordinate representation of all operators, instead of the energy representation used in the previous section. The partition function is now written as:

$$
Z=\int d q\langle q|\exp (-\beta \mathcal{H C})| q\rangle
$$

where $q$ stands for the coordinates $q_{1}, \cdots, q_{N}$ of all the particles and where, if the wall potential is included in $\mathfrak{H}$, the integrals may be taken from $-\infty$ to $+\infty$. Introducing the reduced coordinates $\bar{q}_{i}=q_{i} L^{-1}$, one finds :

$$
\begin{aligned}
V \frac{\partial Z}{\partial V}=\frac{L}{3} \frac{\partial Z}{\partial L} & =N Z \\
& +\frac{L^{3 N}}{3} \int d \tilde{q} L \frac{\partial}{\partial L}\langle L \bar{q}|\exp (-\beta \mathcal{H})| L \bar{q}\rangle .
\end{aligned}
$$

To carry out the differentiation, one expands the matrix $\exp (-\beta \mathfrak{H C})$ in its power series in $\mathfrak{H C}$, and then differentiates term by term. Following Green, one obtains for the first term, going back to the original variables $q_{i}$ after the differentiation is completed:

$$
\begin{aligned}
& L \frac{\partial}{\partial L}\left\langle L \bar{q}|\mathcal{H}| L \bar{q}^{\prime}\right\rangle \\
& =\left(q \cdot \frac{\partial}{\partial q}+q^{\prime} \cdot \frac{\partial}{\partial q^{\prime}}\right)\left\langle q|\mathcal{H}| q^{\prime}\right\rangle+\left\langle q\left|\Phi_{\mathrm{wall}}\right| q^{\prime}\right\rangle
\end{aligned}
$$

$$
\Phi_{\mathrm{w} \text { all }}=-\sum_{i} q_{i} \frac{\partial W}{\partial q_{i}}
$$

is the virial of the wall potential. Analogously, one can proceed in the differentiation of the matrix elements of the higher powers of $\mathfrak{K C}$, and one can show by induction:

$$
\begin{aligned}
L \frac{\partial}{\partial L}\left\langle L \bar{q}|\exp (-\beta \mathcal{H C})| L \bar{q}^{\prime}\right\rangle \\
=\left(q \cdot \frac{\partial}{\partial q}+q^{\prime} \cdot \frac{\partial}{\partial q^{\prime}}\right)\left\langle q|\exp (-\beta \mathcal{H C})| q^{\prime}\right\rangle \\
\quad-\sum_{m=0}^{\infty} \frac{\beta^{m+1}}{(m+1) !}\left\langle q\left|\mu_{m} \exp (-\beta \mathcal{H C})\right| q^{\prime}\right\rangle,
\end{aligned}
$$

where $\mu_{0} \equiv \Phi_{W}$, and $\mu_{m}=\left[\mu_{m-1}, \mathfrak{F}\right]$, and the square brackets indicate as usual the commutator. Introducing (11) in (10) and using (1), one easily verifies:

$$
\begin{aligned}
& p=\frac{i}{3 \beta h V Z} \int d q\langle q|[p \cdot q, \exp (-\beta \mathfrak{H})]| q\rangle \\
& \quad-\frac{1}{3 V Z} \sum_{m=0}^{\infty} \frac{\beta^{m}}{(m+1) !} \int d q\left\langle q\left|\mu_{m} \exp (-\beta \mathcal{C})\right| q\right\rangle .
\end{aligned}
$$

The first term in (12) is identical with Green's expression for the pressure. The reason that he does not obtain the second term in (12) is clearly due to the fact that he does not include the wall potential in the Hamiltonian, but instead restricts his coordinates $q_{i}$ to the finite range $0 \leqslant q_{i} \leqslant L$. Omitting the wall potential, the matrix element of $\exp (-\beta \mathcal{K})$ in the coordinate representation is apparently a function of $q, q^{\prime}$ alone. Therefore with the reduced coordinates the differentiation operation $L(\partial / \partial L)$ is apparently equivalent to the differentiation operation $q \cdot(\partial / \partial q)+q^{\prime} \cdot\left(\partial / \partial q^{\prime}\right)$ on the original matrix element. The second term in (11) would not be there and one would obtain Green's result for the pressure. We do not believe that this is correct, because the matrix element of $\exp (-\beta \mathcal{H C})$ besides being a function of $q, q^{\prime}$ is also implicitly a function of $L$ because of the boundary condition imposed on the wave functions. One can see this, for instance, by going back to the energy representation. One has:

$$
\left\langle q|\exp (-\beta \mathcal{H C})| q^{\prime}\right\rangle=\sum_{n} \exp \left(-\beta E_{n}\right) \Psi_{n}^{*}(q) \Psi_{n}\left(q^{\prime}\right) .
$$

Following Green, one would neglect, for instance, the dependence of $E_{n}$ on $L$. This, however, just gives, as shown in the previous section, De Boer's result. The other terms (including those of Green) obtained from the $L(\partial / \partial L)$ differentiation must, therefore, be zero. This is in fact, we think, the case. Contrary to Green, and in accordance with De Boer, we are of the opinion that the traces of the commutators which occur in the usual applications of quantum statistics and in particular in (12) are all zero, $\dagger$ so that (12) reduces to the trivial result:

$$
p V=-\frac{1}{3}\left\langle\left(\Phi_{\text {wall }}\right)_{n}\right\rangle_{\mathrm{Av}} \text {. }
$$

In order to obtain (2) from (9) one must carry out the $L$-differentiation more directly, essentially as De Boer does. Since

and

$$
\left\langle q|\exp (-\beta \mathcal{H C})| q^{\prime}\right\rangle=\exp \left(-\beta \mathcal{H}_{\mathrm{op}}\right) \delta\left(q-q^{\prime}\right)
$$

$$
\delta\left(L \bar{q}-L \bar{q}^{\prime}\right)=1 / L \delta\left(\bar{q}-\bar{q}^{\prime}\right),
$$

one can write for the partition function

$$
Z=\iint d \bar{q} d \bar{q}^{\prime} \delta\left(\bar{q}-\bar{q}^{\prime}\right) \exp \left(-\beta \mathcal{H}_{\mathrm{op}}\right) \delta\left(\bar{q}-\bar{q}^{\prime}\right)
$$

† For some further comments on this point see Section 5. This is the main point of the criticism which Green made against De Boer's method of proof. 
Now $L$ appears only in $\Re_{\text {op }}$ (compare Eq. (7)), and one proves that

$$
L \frac{\partial}{\partial L} \exp \left(-\beta \mathcal{K}_{\mathrm{op}}\right)=-\sum_{m=0}^{\infty} \frac{\mu_{m} \beta^{m+1}}{(m+1) !} \exp \left(-\beta \mathcal{H C}_{\mathrm{op}}\right),
$$

where again $\mu_{m}=\left[\mu_{m-1}, \mathfrak{K}\right]$, but where now

$$
\mu_{0} \equiv L \frac{\partial \mathcal{H}_{\mathrm{op}}}{\partial L}=-2 K-\Phi
$$

according to (8). $\dagger \dagger$ Omitting the traces of all commutators, this leads immediately to (2). I

\section{ON THE TRACES OF COMMUTATORS IN QUANTUM STATISTICS}

Green remarks that the trace of the commutator of two matrices $A$ and $B$ is not necessarily zero, since it depends on the convergence of the double integral (or double sum):

$$
\iint d q^{\prime} d q^{\prime \prime}\left\langle q^{\prime}|A| q^{\prime \prime}\right\rangle\left\langle q^{\prime \prime}|B| q^{\prime}\right\rangle
$$

and this may not be the case if $A$ and $B$ are singular, as, for instance, $p$ and $q$. However, in quantum statistics one of the matrices occurring is always the density matrix :

$$
\begin{aligned}
p\left(q, q^{\prime}\right)=\left\langle q|\exp (-\beta 3 \mathcal{C})| q^{\prime}\right\rangle & \\
& =\sum_{n} \exp \left(-\beta E_{n}\right) \Psi_{n}{ }^{*}(q) \Psi_{n}\left(q^{\prime}\right) .
\end{aligned}
$$

For the usual types of Hamiltonians $\rho\left(q, q^{\prime}\right)$ is an analytic function of $q$ and $q^{\prime}$, vanishing exponentially for large values of $q$ and $q^{\prime}$. This follows from the fact

\footnotetext{
If Equation (14) is equivalent to $(I)$ in De Boer's paper after a slight error is corrected.

T(13) would result if one carries the $L$-differentiation out without introducing the reduced coordinates $\bar{q}_{i}$. One can then use (14) again, but now

$$
\mu_{0}=L(\partial W / \partial L)=+\Phi_{\text {wall }}
$$
}

that $\rho\left(q, q^{\prime}\right)$ is the fundamental solution of the parabolic differential equation: $\|$

$$
\frac{\partial \varphi(q, \beta)}{\partial \beta}=-\mathfrak{K}_{\mathrm{op} \varphi}=\frac{\hbar^{2}}{2 m} \Delta \varphi-V \varphi .
$$

For instance, for the harmonic oscillator with

$$
\begin{aligned}
\rho\left(q, q^{\prime}\right) & =\left\{\frac{\omega}{2 \pi \hbar \sinh (\hbar \omega \beta)}\right\}^{\frac{1}{2}}\left(p^{2}+\omega^{2} q^{2}\right): \\
& \exp \left[-\frac{\omega}{2 \hbar[1-\exp (-2 \hbar \omega \beta)]}\right. \\
& \left.\left\{\left(q^{2}+q^{\prime 2}\right)(1+\exp (-2 \hbar \omega \beta))-4 q q^{\prime} \exp (-\hbar \omega \beta)\right\}\right]
\end{aligned}
$$

For such functions we believe that the integrals of the type (15) are always convergent, and therefore the order of integration may be interchanged, as is necessary in order to prove that the trace of the commutator is zero. For example:

$$
\begin{aligned}
\operatorname{Tr}[p, \exp (-\beta \mathcal{C})] & =\frac{\hbar}{i} \int_{-\infty}^{\infty} d q\left(\frac{\partial \rho}{\partial q}+\frac{\partial \rho}{\partial q^{\prime}}\right)_{q=q^{\prime}} \\
& =\frac{\hbar}{i} \int_{C}\left[\frac{\partial \rho}{\partial q} d q+\frac{\partial \rho}{\partial q^{\prime}} d q^{\prime}\right],
\end{aligned}
$$

where $C$ is the path $q=q^{\prime}$ from $-\infty$ to $+\infty$. The line integral is clearly zero since $\rho$ is zero at infinity. Analogously, one shows that the trace of the commutator of $p^{2}$ and of $p \cdot q$ with $\exp (-\beta \pi)$ is zero. In general, the theorems:

$$
\begin{aligned}
\operatorname{Tr}(A B) & =\operatorname{Tr}(B A) \\
\operatorname{Tr}(A B C) & =\operatorname{Tr}(C A B)=\operatorname{Tr}(B C A)
\end{aligned}
$$

and so on, will be valid if only one of the matrices $A$, $B, C, \cdots$ is the density matrix. The other matrices may then be of the singular kind as, for example, the impulse and coordinate matrices are.

|| This means that $\rho\left(q, q^{\prime}\right)$ is the solution of $(16)$, which for $\beta=0$ becomes $\delta\left(q-q^{\prime}\right)$. Of course, certain regularity conditions are necessary for $V(q)$. 